of Lemma 3 to obtain suitable $\theta$ 's for groups of the form $Z_{1} \times Z_{2} \times Z_{3}$ where $Z_{i}$ are cyclic of order $2^{n} i$. However, it should be noted that if $G \cong G_{1} \times G_{2}$, a one-to-one mapping $\theta$ of $G$ upon $G$ may be defined by

$$
\theta[(x, y)]=\left[\theta_{1}(x), \theta_{2}(y)\right]
$$

where $\theta_{1}$ and $\theta_{2}$ are one-to-one mappings of $G_{1}$ upon $G_{1}$ and $G_{2}$ upon $G_{2}$ respectively. Moreover $\theta$ satisfies the relationship $O(\eta) \geqq O\left(\eta_{1}\right) \cdot O\left(\eta_{2}\right)$. Thus if $O\left(\eta_{1}\right)=n\left(G_{1}\right), O\left(\eta_{2}\right)=n\left(G_{2}\right)$ we would have $O(\eta)=n\left(G_{1} \times G_{2}\right)$ and $\theta$ is represented explicitly in terms of $\theta_{1}$ and $\theta_{2}$.

UNIVERSITY OF WISCONSIN

\title{
ON RINGS WHOSE ASSOCIATED LIE RINGS ARE NILPOTENT
}

\section{S. A. JENNINGS}

1. Introduction. With any ring $R$ we may associate a Lie ring $(R)_{l}$ by combining the elements of $R$ under addition and commutation, where the commutator $x \circ y$ of two elements $x, y \in R$ is defined by

$$
x \circ y=x y-y x \text {. }
$$

We call $(R)_{l}$ the Lie ring associated with $R$, and denote it by $\Re$. The question of how far the properties of $\Re$ determine those of $R$ is of considerable interest, and has been studied extensively for the case when $R$ is an algebra, but little is known of the situation in general. In an earlier paper the author investigated the effect of the nilpotency of $\Re$ upon the structure of $R$ if $R$ contains a nilpotent ideal $N$ such that $R / N$ is commutative. ${ }^{1}$ In the present note we prove that, for an arbitrary ring $R$, the nilpotency of $\Re$ implies that the commutators of $R$ of the form $x \circ y$ generate a nil-ideal, while the commutators of $R$ of the form ( $x \circ y) \circ z$ generate a nilpotent ideal (cf. $\S 3$ ). If $R$ is finitely generated, and $\Re$ is nilpotent then the ideal generated by the commutators $x \circ y$ is also nilpotent (cf. $\$ 4$ ).

2. A lemma on $L$-nilpotent rings. We recall that the Lie ring $\Re$ is said to be nilpotent of class $\gamma$ if we have

Received by the editors December 23, 1946.

1 Central chains of ideals in an associative ring, Duke Math. J. vol. 9 (1942) pp. 341-355, Theorem 6.5. 


$$
\Re=\Re_{1} \supset \Re_{2} \supset \cdots \supset \Re_{\gamma} \supset \Re_{\gamma+1}=0
$$

where $\Re_{k}=\left[\Re_{k-1}, \Re\right]$ is the Lie ideal of $\Re$ generated by all elements of the form $x \circ y$ with $x \in \Re$ and $y \in \Re_{k-1}$. If $R$ is a ring whose associated Lie ring is nilpotent of class $\gamma$ then we shall say that $R$ is $L$-nilpotent of class $\gamma$. It is well known that the lower central chain (1) has the property $\left[\Re_{\lambda}, \Re_{\mu}\right] \subseteq \Re_{\lambda+\mu}$ and hence in particular

$$
\left[\Re_{\lambda}, \Re_{\lambda}\right]=0 \quad \text { if } 2 \lambda>\gamma .
$$

We prove the following lemma.

LEMMA 1. Let $R$ be an L-nilpotent ring of class $\gamma$. If $c \in \Re_{\gamma-1}$ and if $x, y$ are arbitrary elements of $R$ then

$$
(c \circ x)(c \circ y)=0 \text {, }
$$

and in particular

$$
(c \circ x)^{2}=0 .
$$

If $c_{1}, c_{2} \in \Re_{\gamma-1}$ and $c_{1} \circ c_{2}=0$ then for arbitrary $x, y \in R$

$$
\left(c_{1} \circ x\right)\left(c_{2} \circ y\right)=0 \text {. }
$$

Proof. Consider the identity

$(\overline{a \circ b y} \circ x)=(\overline{a \circ b} \circ x) y+(a \circ b)(y \circ x)+b(\overline{a \circ y} \circ x)+(b \circ x)(a \circ y)$.

Setting $a=b=c$ we have, since $\left[\Re_{\gamma-1}, \Re, \Re\right]=0$,

$$
0=(c \circ x)(c \circ y)
$$

and, if $x=y$,

$$
0=(c \circ x)^{2}
$$

while if $a=c_{2}, b=c_{1}$ and $c_{1} \circ c_{2}=0$

$$
0=\left(c_{1} \circ x\right)\left(c_{2} \circ y\right),
$$

which proves the lemma.

3. Ideals generated by the lower central chain of $\Re$. In what follows, $R$ will be an $L$-nilpotent ring, and we denote the lower central chain of $\Re$ as in (1). Let $R_{k}, k=1,2, \cdots, \gamma$, be the subring of $R$ generated by the elements of $\Re_{k}$, and let $\bar{R}_{k}$ be the ideal of $R$ generated by $R_{k}$. It is known ${ }^{2}$ that every element of $\bar{R}_{k}$ may be written in the form $u_{k}+v_{k}$, where $u_{k} \in R_{k}$ and $v_{k} \in R R_{k}$, and since $R_{\gamma}$ is in the centre of $R, \bar{R}_{\gamma}$ is a nilpotent or nil-ring whenever $R_{\gamma}$ is.

2 Ibid. Lemma 5.3. 
Let $R^{*}=R / \bar{R}_{\gamma}$; then the natural homomorphism of $R$ upon $R^{*}$ induces a homomorphism of $\Re$ upon $\Re^{*}$, where $\Re^{*}$ is the Lie ring associated with $R^{*}$, such that $\bar{R}_{k} \rightarrow \bar{R}_{k}^{*}$. Hence in particular $\Re_{\gamma}^{*}=0$ and $R^{*}$ is an $L$-nilpotent ring of class not greater than $\gamma-1$.

Our principal theorem is the following:

THEOREM 1. If $R$ is an L-nilpotent ring, then the commutators of $R$ generate a nil-ideal of $R$, that is, $\bar{R}_{2}$ is a nil-ideal. The elements of $R$ of the form $(x \circ y) \circ z$ generate a nilpotent ideal of $R$, that is, $\bar{R}_{3}$ is nilpotent.

Proof. Consider first $R_{\gamma}$ : every element of $R_{\gamma}$ can be written as a finite sum of finite products of elements of $\Re_{\gamma}$ and since $\Re_{\gamma}=\left[\Re_{\gamma-1}, \Re\right]$, every element of $\Re_{\gamma}$ can be written as a finite sum of elements of the form $c \circ x$, where $c \in \Re_{\gamma-1}$ and $x \in R$. Hence every element of $R_{\gamma}$ is a sum of products of elements of the form $c \circ x$. Now by Lemma 1 the square of every element of the form $c \circ x$ is zero, and these elements are all in the centre of $R$. Hence if

$$
y=p_{1}+p_{2}+\cdots+p_{n}
$$

is an element of $R_{\gamma}$, where the $p_{k}$ are products of elements of the form $c \circ x$, we have $p_{k}^{2}=0$ and therefore, since these products $p_{k}$ are all in the centre of $R$, we have $y^{n+1}=0$, which proves that $R_{\gamma}$, and hence $\bar{R}_{\gamma}$, is a nil-ring. Now if $\gamma>2$ we have, from (2)

$$
\left[\Re_{\gamma \rightarrow 1}, \Re_{\gamma-1}\right]=0
$$

and hence $c_{1} \circ c_{2}=0$ for all $c_{1}, c_{2} \in \Re_{\gamma-1}$. From Lemma 1 it follows that $p_{i} p_{j}=0$ in the representation of $y$ above, and hence

$$
\bar{R}_{\gamma}^{2}=0 \text {, }
$$

The proof of the theorem now proceeds easily by induction upon $\gamma$, since by the above it is true when $\gamma=2$, that is whenever $\bar{R}_{2}=\bar{R}_{\gamma}$, $\bar{R}_{\mathbf{3}}=0$. We suppose, therefore, that the theorem holds for rings of class less than $\gamma$, and hence in particular for $R^{*}=R / \bar{R}_{\gamma}$. Then if $c \in \bar{R}_{2}$ and $c \rightarrow c^{*}$ in the homomorphism of $R$ upon $R^{*}$ we have

$$
c^{* \sigma^{\prime}}=0, \quad \sigma^{\prime} \text { some integer, }
$$

by our induction, and hence

$$
c^{\sigma^{\prime}} \in \bar{R}_{\gamma} \quad \text { for all } c \in \bar{R}_{2 \bullet}
$$

Since $\bar{R}_{\gamma}^{2}=0$ whenever $\gamma>2$ we have

$$
c^{\sigma}=0, \quad \text { where } \sigma=2 \sigma^{\prime},
$$


and it follows that $\bar{R}_{2}$ is a nil-ring. Further, since $\bar{R}_{\mathbf{3}}{ }^{*}$ is nilpotent by our induction,

$$
\bar{R}_{3}^{* *^{\prime}}=0 \quad \text { for some integer } \tau^{\prime}
$$

and hence

$$
\bar{R}^{\tau^{\prime}} \subseteq \bar{R}_{\gamma}
$$

and therefore

$$
\bar{R}_{3}^{r}=0, \quad \quad \text { where } \tau=2 \tau^{\prime},
$$

which proves that $\bar{R}_{3}$ is nilpotent, as required.

4. Finitely generated $L$-nilpotent rings. If $R$ satisfies the maximal or minimal condition for one-sided ideals, so does $\bar{R}_{2}$ and hence $\bar{R}_{2}$ must be nilpotent. ${ }^{3}$ We prove the following stronger result:

THEOREM 2. If $R$ is a finitely generated L-nilpotent ring, then the commutators of $R$ generate a nilpotent ideal, that is, $\bar{R}_{2}$ is nilpotent.

Proof. If $R$ is finitely generated, say by $x_{1}, x_{2}, \cdots, x_{d}$, then every element $x$ of $R$ can be written in the form $x=p_{1}+p_{2}+\cdots+p_{n}$ where the $p_{k}$ are products of the $x_{1}, \cdots, x_{d}$ in some order. It is clearly sufficient to consider the case $\gamma=2$, since if we show in general that $\bar{R}_{2} / \bar{R}_{3}$ is nilpotent, it will follow from Theorem 1 that $\bar{R}_{2}$ has this property. Because of the identity

$$
(a b) \circ c=a(b \circ c)+(a \circ c) b
$$

every element of $\bar{R}_{2}$ can be written as a sum of products of the form

$$
\pi_{r}=a\left(x_{i_{1}} \circ x_{j_{1}}\right)\left(x_{i_{2}} \circ x_{j_{2}}\right) \cdots\left(x_{i_{r}} \circ x_{j_{r}}\right), \quad a \in R .
$$

Now there are at most $d(d-1) / 2$ nonzero commutators of the type $x_{i} \circ x_{j}$, and since by Lemma 1 we have

$$
\left(x_{i} \circ x_{j}\right)\left(x_{i} \circ x_{k}\right)=0
$$

it follows that if the number of factors in any product $\pi_{r}$ is greater than $d(d-1) / 2$ this product vanishes. Hence

$$
\bar{R}_{2}^{\tau}=0, \quad \tau=d(d-1) / 2+1
$$

and the theorem is established.

- Cf. C. Hopkins, Nilrings with minimum condition for admissible left ideals, Duke Math. J. vol. 4 (1938) pp. 664-667; J. Levitzki, Solution of a problem of G. Köthe, Amer. J. Math. vol. 67 (1945) pp. 437-442. 
In connection with Theorem 2 it would be of interest to know if there exist $L$-nilpotent rings for which $R_{\mathbf{2}}$ is not nilpotent. It would be enough to exhibit a ring $R$ for which

$$
(x \circ y) \circ z=0 \quad \text { for all } x, y, z \in R
$$

and such that the subring generated by elements of the form $(x \circ y)$ is not nilpotent. The author has been unable to construct such a ring but it seems fairly safe to conjecture that such a one exists, and indeed with a countable generating set.

Since $R / \bar{R}_{2}$ is commutative and $\bar{R}_{2}$ is nilpotent we have at once from an earlier result of the author $:^{1}$

Theorem 3. A finitely generated L-nilpotent ring is of finite class.

Finally, it is clear that we have the following criterion for the nilpotency of a finitely generated nil-ring:

THEOREM 4. A finitely generated nil-ring is nilpotent if and only if its associated Lie ring is nilpotent.

This last theorem may be compared with Kaplansky's result on finitely generated nil-algebras, ${ }^{4}$ which states that, provided the ground field has enough elements, such an algebra is nilpotent if and only if there exists a fixed integer $\rho$ such that $x^{\rho}=0$ for all elements $x$ in the algebra. Our theorem shows that this condition may be replaced by the requirement that all commutators of a fixed weight vanish.

The University of British Columbia

'I. Kaplansky, On a problem of Kurosch and Jacobson, Bull. Amer. Math. Soc. vol. 52 (1946) pp. 496-500. Added in proof. In a recent paper (Bull. Amer. Math. Soc. vol. 52 (1946) pp. 1033-1035) J. Levitzki has proved a more general theorem to the effect that every finitely generated nil-ring of bounded index is nilpotent. 